\title{
2. SYNOPSIS
}

\begin{tabular}{|l|l|}
\hline Name of Sponsor: I.R.I.S., 50 rue Carnot - 92284 Suresnes Cedex - France & (For National \\
\hline Test drug & \\
Name of Finished Product: & \\
Valdoxan® & \\
Name of Active Ingredient: & \\
Agomelatine (S 20098) & Page: \\
\hline Individual Study Table Referring to Part of the Dossier & Volume: \\
\hline
\end{tabular}

Title of study: Efficacy and safety of agomelatine ( $25 \mathrm{mg} /$ day with blinded potential adjustment to $50 \mathrm{mg} / \mathrm{day})$ for 12 weeks in non-depressed out-patients with Generalized Anxiety Disorder.

A 12-week randomised, double-blind, versus venlafaxine XR $(75 \mathrm{mg} /$ day with blinded potential adjustment up to $225 \mathrm{mg}$ /day), 2-arm parallel groups, international multicenter study.

Protocol No.: CL3-20098-078

ISRCTN Register: ISRCTN75061806

The description of the study protocol given hereafter includes the modifications of the 2 substantial amendments to the protocol.

National coordinators:

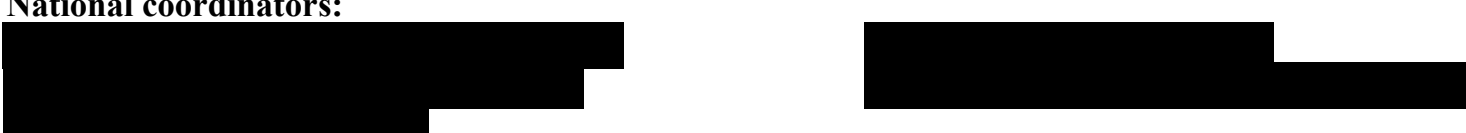

\section{Study centres:}

In all, 24 centres located in 5 countries/regions included at least one patient: China (10 centres - 148 patients), Taiwan ( 4 centres -16 patients), Malaysia ( 6 centres -15 patients), Singapore ( 1 centre -1 patient), Thailand ( 3 centres -7 patients).

Publication (reference): Not Applicable

Studied period:

Initiation date: 24 December 2012 (date of first visit first patient)

Completion date: 12 February 2014 (date of last visit last patient)

\section{Objectives:}

The primary objective of this study was to assess the efficacy of agomelatine (25-50 mg/day p.o.) compared to venlafaxine extended release (XR) (75-150-225 mg/day p.o.) after a 12-week treatment in non-depressed out-patients suffering from Generalized Anxiety Disorder (GAD).

The secondary objectives were to further describe the effect on anxiety symptoms, sleep patterns and social functioning, and to provide additional safety data on agomelatine in Asian population.

\section{Methodology:}

Phase III, international, multicentre, double-blind, randomized 12-week study with two-arm parallel group (agomelatine 25-50 mg versus venlafaxine XR 75-150-225 mg) in non depressed patients suffering from Generalized Anxiety Disorder.

Dose adjustment was possible at W4 (for both treatments) and W6 (for patients on venlafaxine $150 \mathrm{mg} / \mathrm{day}$ ). The criteria for increasing the dose were defined by the sponsor, based on clinical considerations, before the study beginning, and kept blinded to the investigator and to the patient. The randomisation was balanced (non-adaptive), with stratification on the centre. The randomisation, the treatment allocation and the dose increase were done centrally using an Interactive Response System (IRS).

This study was performed in strict accordance with Good Clinical Practice including the archiving of essential documents.

The study was prematurely stopped following a sponsor's strategic decision.

Number of patients:

Planned: 506 patients included, i.e. 253 patients by treatment group.

Included: 187 patients (37.0\% of the number planned): 96 patients in the agomelatine group, 91 patients in the venlafaxine XR group, following an internal decision of stopping the study earlier. 


\section{Diagnosis and main criteria for inclusion:}

Asian adult (legal age for majority) out-patients of both genders, fulfilling DSM-IV-TR criteria for Generalized Anxiety Disorder, confirmed by M.I.N.I. questionnaire and requiring a psychotropic treatment.

At selection, HAM-A total score was to be $\geq 22$, HAM-A item 1 (anxious mood) $\geq 2$ and item 2 (tension) $\geq 2$, HAM-A item $1+$ item $2 \geq 5$, HAD anxiety sub-score $\geq$ HAD depression sub-score, and MADRS total score was to be $\leq 16$. At inclusion HAM-A total score was still to be $\geq 22$ and no more than a $20 \%$ of decrease (if any) in HAM-A total score between selection and inclusion, HAD anxiety sub-score was still to be $\geq$ HAD depression sub-score, and the MADRS total score was still to be $\leq 16$.

\section{Test drug:}

Agomelatine encapsulated tablets $25 \mathrm{mg}$ or $50 \mathrm{mg}, 1$ capsule per day taken orally in the evening.

Patients received $25 \mathrm{mg}$ /day from W0 with possible increase to $50 \mathrm{mg} /$ day in double-blind conditions from W4, in case of insufficient improvement. Once adjusted (or not) at W4, the dose was maintained up to W12. At W12, or in case of premature withdrawal from W4, patients received the dose received from W4 for additional 14 days (corresponded to the tapering period for venlafaxine recommended to avoid possible withdrawal reactions).

Batch Nos. L0047440, L0044537, L0048179, L0044753, L0049420.

\section{Comparator:}

Venlafaxine XR encapsulated capsules $75 \mathrm{mg}$ or $150 \mathrm{mg}$ or $225 \mathrm{mg}, 1,2$ or 3 capsules once daily taken orally in the morning with breakfast.

Patients received $75 \mathrm{mg}$ /day from W0, with possible increase in double-blind conditions to $150 \mathrm{mg} / \mathrm{day}$ at W4, in case of insufficient improvement. At W6, the patients receiving $150 \mathrm{mg} /$ day and not sufficiently improved could receive $225 \mathrm{mg}$ /day. Once adjusted, the dose was maintained up to W12. At W12, or in case of premature withdrawal from W4, patients having previously received venlafaxine XR $225 \mathrm{mg} /$ day received $150 \mathrm{mg}$ /day between $\mathrm{W} 12$ and $\mathrm{W} 13$, then $75 \mathrm{mg} / \mathrm{d}$ between $\mathrm{W} 13$ to $\mathrm{W} 14$. The patients having previously received venlafaxine XR $150 \mathrm{mg}$ /day received $75 \mathrm{mg}$ /day between $\mathrm{W} 12$ and $\mathrm{W} 14$ (tapering period recommended to avoid possible withdrawal reactions).

Batch Nos. L0047640, L0044536, L0044556, L0048162.

\section{Duration of treatment:}

- Run-in period: 1-2 week(s) without treatment (from selection to W0).

- Active treatment period: 12 weeks (from W0 to W12).

- Tapering Phase: 2 weeks (from W12 to W14).

- Follow-up period: 1 week after discontinuation of study treatment (after W14 or after treatment discontinuation).

\section{Criteria for evaluation:}

\section{Efficacy measurements:}

- Hamilton Anxiety Rating Scale (HAM-A) was rated by the investigator at each visit from the selection visit to W12. The primary efficacy criterion was the HAM-A total score. The main analytical approach was the change from baseline to last post-baseline value over the W0-W12 period.

- Clinical Global Impressions scale (CGI) was rated by the investigator at each visit from the selection visit (at selection and inclusion visits, only severity of illness score (CGI-S)) to W12.

- Hospital Anxiety and Depression scale (HAD) was completed by the patient at selection, inclusion, W8, and W12, or in case of premature withdrawal.

- Leeds Sleep Evaluation Questionnaire (LSEQ) was completed by the patient at W2, W4, and W12 or in case of premature withdrawal.

- Sheehan Disability Scale (SDS) was completed by the patient at selection, W4, W8, and W12, or in case of premature withdrawal. 


\section{Criteria for evaluation (Cont'd):}

\section{Safety measurements:}

- Adverse events reported at each visit.

- Laboratory tests (haematology, biochemistry): blood samplings were prescribed at selection, at W2 (only liver function parameters), at W6 and W12 in order to have the results at W0, W4, W8, and W14, respectively, or in case of withdrawal in order to have the results at Wend.

- Physical examination: vital signs, i.e. sitting blood pressure (DBP, SBP) and heart rate (HR) were measured at each visit from selection up to W14, or in case of premature withdrawal. Body weight was measured at selection, inclusion, W4, and W12, or in case of premature withdrawal. Height was measured at selection.

- 12-lead ECGs: one ECG was prescribed at selection in order to have the result for inclusion, and at W12 in order to have the result at W14, or in case of withdrawal in order to have the result for Wend.

- Liver B ultrasound detection (only for patients of China mainland) was prescribed before the inclusion of the patient in the study and at W12 in order to have the results at W0, and W14, respectively, or in case of withdrawal in order to have the results at Wend.

- Columbia-Suicide Severity Rating Scale (C-SSRS) (added by amendment No. 1) was rated by the investigator at W0 (baseline version) and at each visit from W2 to Wend (since last visit version).

- Psychotropic-Related Sexual Dysfunction Questionnaire (PRSexDQ) was rated by the investigator at W0, W4 and W12.

\section{Other criteria not specifically related to efficacy or safety:}

- Health related quality of life: EuroQol Questionnaire (EQ-5D) was completed by the patient at W0, W2, W4, W8, W12 and Wend.

- Socioeconomic evaluation: Self-rating questions were completed at W0, W4, W12 and Wend.

\section{Statistical methods:}

\section{Analysis Set:}

Efficacy analyses were performed in the Full Analysis Set (FAS) defined (in accordance with the intention-to-treat principle and ICH-E9 guideline) as all patients of the RS having taken at least one dose of study medication and having a value at baseline and at least one post-baseline value for the primary efficacy criterion on the W0-W12 period.

\section{Efficacy analysis:}

\section{Primary criterion: HAM-A total score}

In addition to descriptive statistics for all analytical approaches over the W0-W12 period, the following analysis was performed:

Main analysis:

The difference in the change from baseline to last post-baseline value between agomelatine and venlafaxine was estimated using a two-way analysis of covariance (ANCOVA) model, on factor treatment as fixed effect with centre as random class effect and baseline HAM-A total score as continuous covariate and without interaction, mixed model.

Secondary analyses:

The response to treatment (defined as a decrease from baseline $\geq 50 \%$ in HAM-A total score) was described by treatment group at each visit and for the last post-baseline value over the W0-W12 period.

Secondary criteria:

For all analytical approaches of secondary criteria, descriptive statistics were provided over the W0-W12 period by treatment group.

\section{Safety analysis:}

For every safety measurement, descriptive statistics were provided in the Safety Set (and in the Sub-SS of HAM-A responders patients for PRSexDQ criteria) and by treatment group. 


\section{SUMMARY - CONCLUSIONS \\ DISPOSITION OF PATIENTS AND ANALYSIS SETS}

\begin{tabular}{|c|c|c|c|c|}
\hline Status & & Agomelatine & Venlafaxine & All \\
\hline Included and randomised & $\mathbf{n}$ & 96 & 91 & 187 \\
\hline Lost to follow-up & n (\%) & - & - & - \\
\hline Withdrawn over W0-W12 due to & n (\%) & $25(26.0)$ & $24(26.4)$ & $49(26.2)$ \\
\hline Adverse event & $\mathrm{n}(\%)$ & $5(5.2)$ & $6(6.6)$ & $11(5.9)$ \\
\hline Protocol deviation & $\mathrm{n}(\%)$ & $1(1.0)$ & $1(1.1)$ & $2(1.1)$ \\
\hline Lack of efficacy & $\mathrm{n}(\%)$ & $3(3.1)$ & $2(2.2)$ & $5(2.7)$ \\
\hline Non-medical reason & $\mathrm{n}(\%)$ & $16(16.7)$ & $15(16.5)$ & $31(16.6)$ \\
\hline Completed the W0-W12 period & n $(\%)$ & $71(74.0)$ & $67(73.6)$ & $138(73.8)$ \\
\hline Entered the W12-W14 period & n (\%) & $71(74.0)$ & $67(73.6)$ & $138(73.8)$ \\
\hline Withdrawn over W12-W14 due to & n (\%) & $1(1.0)$ & $2(2.2)$ & 3 (1.6) \\
\hline Adverse event & $\mathrm{n}(\%)$ & $1(1.0)$ & $1(1.1)$ & $2(1.1)$ \\
\hline Non medical reason & $\mathrm{n}(\%)$ & - & $1(1.1)$ & $1(0.5)$ \\
\hline Completed the W12-W14 period & n (\%) & $70(72.9)$ & $65(71.4)$ & $135(72.2)$ \\
\hline Full Analysis Set & n (\%) & $89(92.7)$ & $78(85.7)$ & $167(89.3)$ \\
\hline Safety Set & n (\%) & 93 (96.9) & $88(96.7)$ & $181(96.8)$ \\
\hline
\end{tabular}

Following the strategic sponsor's decision to stop the agomelatine GAD development in China, the study was prematurely stopped. A total of 249 patients were screened and 221 were selected for the study. Among them, 187 patients were included; i.e. $37.0 \%$ of patients planned in the protocol. They were randomly assigned to one of the 2 groups: 96 patients in the agomelatine group, and 91 in the venlafaxine group. A balanced distribution between the 2 treatment groups was observed.

Among these patients, $74.0 \%$ and $73.6 \%$ in the agomelatine and venlafaxine groups, respectively, completed the W0-W12 period. All these patients entered the W12-W14 tapering period.

During the W0-W14 period, the rate of study withdrawal was similar in the agomelatine and venlafaxine groups $(27.1 \%$ and $28.6 \%$, respectively). In both treatment groups, the most frequent reasons were non-medical reason ( $16.7 \%$ and $17.6 \%$, respectively), then adverse events (6.3\% and $7.7 \%$, respectively).

\section{BASELINE CHARACTERISTICS}

In the Randomised Set, the patients were aged from 19 to 65 years with a mean \pm SD of $39.4 \pm 11.6$ years. Half patients were female (50.8\%).

All patients fulfilled DSM-IV diagnostic criteria for GAD. The time since GAD diagnosis covered a wide range from 0 to 242 months $(0-20.2$ years). At least $75 \%$ of patients had a GAD diagnosis made within the month before selection. In the same way, the duration since the first anxious symptoms with a functional impact ranged within 6 to 360 months ( 0 - 30 years) with a median duration of 30 months ( 2.5 years).

About three quarters of patients (77.5\%) had not taken any previous psychotropic treatments, i.e. anxiolytics (including benzodiazepines) and/or antidepressants within the last 12 months before the selection. At inclusion, no patient received a specific concomitant treatment.

At inclusion, the mean \pm SD MADRS total score was $11.7 \pm 2.8$ and as required in the selection/inclusion criteria all MADRS total scores were $\leq 16$. The mean HAD depression sub-score was $6.3 \pm 3.8$.

At inclusion, the mean HAM-A total score was $28.5 \pm 4.5$ and as required in the selection/inclusion criteria all HAM-A total scores were $\geq 22$. The mean HAM-A psychic anxiety score was higher than the mean somatic anxiety score (15.7 \pm 2.5 and $12.8 \pm 3.3$, respectively). The mean \pm SD HAD anxiety sub-score was $13.4 \pm 3.3$. The mean \pm SD CGI severity of illness score was $4.7 \pm 0.7$.

At inclusion visit, the mean \pm SD SDS total score and item scores were as follows:

- SDS total score: $16.5 \pm 6.0$.

- Work: $5.8 \pm 2.3$

- Social life: $5.6 \pm 2.3$

- Family life and home responsibilities: $5.2 \pm 2.4$.

There were no clinically relevant differences between the two treatment groups for demographic criteria, GAD characteristics, HAM-A total score, and all anxiety criteria. 


\section{SUMMARY - CONCLUSIONS (Cont'd) \\ EXTENT OF EXPOSURE}

In the Randomised Set, the treatment duration over the W0-W12 period ranged between 0 and 104 days with a mean \pm SD of $73.3 \pm 26.4$ days and a median of 84 days. The mean \pm SD overall compliance was $90.9 \pm 20.9 \%$ for the yellow capsules (agomelatine) and $91.2 \pm 21.2 \%$ for the red capsules (venlafaxine). No relevant differences were observed between the two treatment groups.

\section{EFFICACY RESULTS}

The following efficacy results correspond to analyses performed in the FAS, i.e. $33.0 \%$ of patients calculated for the sample size.

\section{Primary assessment criterion}

HAM-A total score

In the FAS, the mean HAM-A total score decreased over the W0-W12 period in the agomelatine and venlafaxine groups. At the last post-baseline assessment, the mean decrease from baseline was not statistically significantly different between the treatment groups (see Table below).

At the last post-baseline assessment, the percentage of responders (decrease in HAM-A total score of at least $50 \%$ from baseline) was $69.7 \%$ and $74.4 \%$ in the agomelatine and venlafaxine groups, respectively.

HAM-A total score: between-group comparisons at the last post-baseline assessment over the W0-W12 period in the FAS

\begin{tabular}{llcc}
\hline & & $\begin{array}{c}\text { Agomelatine } \\
(\mathbf{N}=\mathbf{8 9})\end{array}$ & $\begin{array}{c}\text { Venlafaxine } \\
(\mathbf{N}=\mathbf{7 8})\end{array}$ \\
\hline W0 & $\mathrm{n}$ & 89 & 78 \\
& Mean \pm SD & $28.2 \pm 4.1$ & $28.7 \pm 4.8$
\end{tabular}

Change from baseline to last post-baseline ${ }_{n}$ assessment

\section{Statistical analysis*}

$$
\text { Mean } \pm \text { SD } \quad-15.8 \pm 8.3 \quad-17.7 \pm 7.6
$$

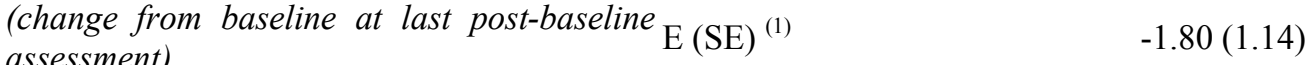

$$
\begin{aligned}
& \text { assessment) } \quad 95 \% \mathrm{CI}^{(2)} \quad[-4.05 ; 0.45]
\end{aligned}
$$

* Main analysis: Analysis of covariance model on factor treatment with centre (random effect) and baseline HAM-A total score as covariates

(1) Estimate (Standard Error) of the difference between adjusted treatment group means: Venlafaxine minus Agomelatine

(2) Two-sided 95\% Confidence Interval of the estimate.

\section{Secondary assessment criteria}

\section{CGI}

In the FAS, at the last (post)-baseline assessment over the W0-W12 period, the mean \pm SD severity of illness and global improvement scores were similar in both treatment groups as follows:

- Severity of illness score: $2.7 \pm 1.2$ and $2.5 \pm 1.3$ in the agomelatine and venlafaxine groups, respectively.

- Global improvement score: $1.9 \pm 1.1$ and $1.7 \pm 1.0$, respectively.

In the same way, the percentage of responders according to CGI global improvement score (score $=1$ or 2$)$ was $78.7 \%$ and $84.6 \%$ at the last assessment, respectively.

\section{HAD}

In the FAS, at last post-baseline assessment, the mean decrease \pm SD in anxiety sub-score from baseline was lower in the agomelatine group $(-6.6 \pm 4.7)$ than in the venlafaxine group $(-8.1 \pm 4.1)$. The mean decrease in depression sub-score from baseline was $-2.1 \pm 3.2$ in the agomelatine group, and $-3.1 \pm 3.7$ in the venlafaxine group. 


\section{SUMMARY - CONCLUSIONS (Cont'd) \\ EFFICACY RESULTS (Cont'd) \\ SDS}

In the FAS, the 3 mean SDS scores rating the disruption of work, social life and family life and home responsibilities, and the SDS total score decreased from baseline to the last post-baseline assessment over the W0-W12 period in the agomelatine and venlafaxine groups indicating an improvement in the 3 functional domains. There were no relevant differences between the treatment groups for all mean decrease scores $\pm \mathrm{SD}$ as follows:

- SDS total score: $-7.4 \pm 6.9$ and $-8.6 \pm 7.1$ in the agomelatine and venlafaxine groups, respectively.

- Work: $-2.7 \pm 2.7$ and $-3.0 \pm 2.6$, respectively.

- Social life: $-2.6 \pm 3.0$ and $-3.1 \pm 2.6$, respectively.

- Family life and home responsibilities: $-2.5 \pm 2.8$ and $-2.7 \pm 2.6$, respectively.

\section{LSEQ}

In the FAS, the 4 mean LSEQ scores showed that the patients had a sleep improvement on average over the W0-W12 period compared to the period without medication in the agomelatine and venlafaxine groups. There were no relevant differences between the treatment groups for all mean scores \pm SD at the last assessment as follows (improvement with lowering score):

- Getting off to sleep score: $35.6 \pm 15.2 \mathrm{~mm}$ and $38.0 \pm 16.7 \mathrm{~mm}$ in the agomelatine and venlafaxine groups, respectively.

- Quality of sleep score: $31.8 \pm 18.4 \mathrm{~mm}$ and $31.8 \pm 20.2 \mathrm{~mm}$, respectively.

- Sleep awakening score: $33.8 \pm 17.6 \mathrm{~mm}$ and $29.9 \pm 18.5 \mathrm{~mm}$, respectively.

- Integrity of behaviour score: $30.7 \pm 20.9 \mathrm{~mm}$ and $26.8 \pm 19.9 \mathrm{~mm}$, respectively.

\section{EQ-5D}

In the FAS, the mean EQ-5D index increased from baseline to the last post-baseline assessment over the W0-W12 period in both treatment groups without relevant difference between them: $0.11 \pm 0.17$ (median 0.06) in the agomelatine group and $0.18 \pm 0.20$ (median 0.19) in the venlafaxine group.

In the same line, the mean increase from baseline in EQ VAS score was $17.0 \pm 18.0$ (median 19.5) in the agomelatine group and $21.3 \pm 17.9$ (median 20.0) in the venlafaxine group at the last post-baseline assessment.

\section{SAFETY RESULTS}

\section{Emergent adverse events}

Over the W0-W14 period in the Safety Set, the percentage of patients who reported at least one emergent adverse event was lower in the agomelatine group than in the venlafaxine group (see below).

\section{Overall summary of adverse events in the Safety Set}

\begin{tabular}{|c|c|c|c|}
\hline & & $\begin{array}{l}\text { Agomelatine } \\
(\mathrm{N}=93)\end{array}$ & $\begin{array}{l}\text { Venlafaxine } \\
\quad(\mathbf{N}=\mathbf{8 8})\end{array}$ \\
\hline \multicolumn{4}{|l|}{ Patients having reported } \\
\hline at least one emergent adverse event & n $(\%)$ & $36(38.7)$ & $53(60.2)$ \\
\hline at least one treatment-related emergent adverse event & $\mathrm{n}(\%)$ & $25(26.9)$ & $43(48.9)$ \\
\hline \multicolumn{4}{|l|}{ Patients having experienced } \\
\hline at least one serious adverse event & $\mathrm{n}(\%)$ & $2(2.2)$ & $3(3.4)$ \\
\hline at least one serious emergent event & $\mathrm{n}(\%)$ & $2(2.2)$ & $2(2.3)$ \\
\hline at least one treatment-related serious adverse event & $\mathrm{n}(\%)$ & - & - \\
\hline \multicolumn{4}{|l|}{ Patients with treatment withdrawal } \\
\hline due to an emergent adverse event & $\mathrm{n}(\%)$ & $6(6.5)$ & $7(8.0)$ \\
\hline due to an emergent serious adverse event & $\mathrm{n}(\%)$ & - & - \\
\hline due a treatment-related emergent adverse event & $\mathrm{n}(\%)$ & $5(5.4)$ & $6(6.8)$ \\
\hline due a treatment-related emergent serious adverse event & $\mathrm{n}(\%)$ & - & - \\
\hline Patients who died & $\mathrm{n}(\%)$ & - & - \\
\hline
\end{tabular}




\section{SUMMARY - CONCLUSIONS (Cont'd) \\ SAFETY RESULTS (Cont'd)}

In the Safety Set, the most frequently affected (at least $5 \%$ of patients) system organ classes in the agomelatine group were gastrointestinal disorders $(17.2 \%$ of patients), skin and subcutaneous tissue disorders $(8.6 \%)$, nervous system disorders (7.5\%), infections and infestations (6.5\%), and investigations (5.4\%).

Among these system organ classes, gastrointestinal disorders and nervous system disorders were less frequently affected in the agomelatine group than in the venlafaxine group $(17.2 \%$ of patients versus $33.0 \%$, respectively, and $7.5 \%$ versus $22.7 \%$, respectively). Conversely, skin and subcutaneous tissue disorders were more frequently affected in the agomelatine group than in the venlafaxine group $(8.6 \%$ of patients versus $2.3 \%)$.

The three most frequent emergent adverse events reported in at least $5 \%$ of patients in the agomelatine group were the same as in the venlafaxine group with a lower frequency in the agomelatine group than in the venlafaxine group (nausea: $7.5 \%$ versus $18.2 \%$, constipation: $6.5 \%$ versus $12.5 \%$ and dizziness: $5.4 \%$ versus $10.2 \%$, respectively). In addition in the venlafaxine group, there were decreased appetite (none in the agomelatine group versus $10.2 \%)$, somnolence $(1.1 \%$ in the agomelatine group versus $6.8 \%)$, and nasopharyngitis (2.2\% in the agomelatine group versus $5.7 \%)$.

The percentage of patients with at least one severe emergent adverse event was lower in the agomelatine group $(2.2 \%, 2$ patients) than in the venlafaxine group (5.7\%, 5 patients).

The percentage of patients with at least one emergent adverse event considered to be related to the study treatment by the investigator was lower in the agomelatine group $(26.9 \%)$ than in the venlafaxine group $(48.9 \%)$.

No death occurred during the study. Two patients in each treatment group (see Table above) had at least one emergent serious adverse event: deep vein thrombosis and myocardial ischaemia in the agomelatine group and haemothorax associated with pneumothorax traumatic, and pneumonia in the venlafaxine group.

None of these events led to study treatment discontinuation. None was considered as related to the study treatment by the investigator and the sponsor. All resolved at the end of the study.

Premature treatment withdrawals were reported in 6 patients $(6.5 \%)$ in the agomelatine group and 7 patients $(8.0 \%)$ in the venlafaxine group without relevant difference between the treatment groups. For all of these patients but two (1 patient in each treatment group), the emergent adverse events leading to the treatment discontinuation were considered as related to the study treatment by the investigator. In the agomelatine group, the type of events leading to treatment discontinuation was disparate except 2 transaminases increased ( 2 patients). In the venlafaxine group, 3 patients stopped the study treatment due to dizziness and 2 patients due to nausea. All patients recovered at the end of the study except one patient in the agomelatine group with an ultrasound liver abnormal.

\section{Laboratory tests}

Emergent PCSA biochemical values were sparse in both treatment groups and for each parameter, except for triglycerides without difference between the two treatment groups $(8.8 \%$ and $8.0 \%$ of patients in the agomelatine and venlafaxine groups, respectively).

One agomelatine-treated patient had PCSA value of ALT (3.9 ULN) after 6 weeks on treatment. This value was associated with AST above the reference limit without reaching PCSA limit (1.9 ULN). No other liver parameters were abnormal. The PCSA value was reported as adverse event and considered as treatment-related by the investigator. The patient recovered 7 days after the treatment withdrawal.

As regards haematological values, 2 PCSA low values of leukocytes were reported in 2 patients in the venlafaxine group. One of these values was reported as adverse event by the investigator, and considered to be not related to the study treatment. The patient recovered on treatment.

\section{Physical examination}

In the Safety Set, there were no relevant mean changes in weight, sitting blood pressures and heart rate between baseline and last post-baseline assessment during the W0-W12 period or W0-W14 period, respectively.

Analysis of BMI by class showed that BMI class increases were little frequent in both treatment groups ( 3 patients, $3.2 \%$ and 2 patients, $2.3 \%$ of patients in the agomelatine and venlafaxine groups, respectively) likewise BMI decreases (5 patients, $5.4 \%$ and 3 patients, $3.4 \%$, respectively). 


\section{SUMMARY - CONCLUSIONS (Cont'd) \\ SAFETY RESULTS (Cont'd)}

\section{ECG}

Among the assessable patients, no emergent ECG abnormality considered clinically significant was reported during the W0-W12 period in both treatment groups.

\section{Columbia-Suicide Severity Rating Scale}

In the Safety Set, no patient prepared, attempted or completed suicide during the W0-W14 period or at Wend in both treatment groups, and no patient had emergent suicidal ideation, non-suicidal self-injury behaviour, suicidal behaviour, or suicidality.

\section{PRSexDQ}

In the Safety Set, the mean PRSexDQ total score at the last post-baseline assessment over the W0-W12 period was similar in both treatment groups $(2.0 \pm 3.1$ and $1.9 \pm 3.0$ in the agomelatine and venlafaxine groups, respectively). No difference was observed between genders.

In the Safety Set, $35.0 \%$ and $36.6 \%$ of patients in the agomelatine and venlafaxine groups, respectively had sexual dysfunctions at the last post-baseline assessment without relevant difference between them. The percentage of patients affected was lower in the agomelatine group than in the venlafaxine group in male patients (29.7\% versus $41.5 \%$, respectively), and inversely in female patients (39.5\% versus $30.0 \%$, respectively). The results of the 4 following items were in the same line:

- Sexual activity change:

- Male: $29.7 \%$ versus $36.6 \%$, respectively.

- Female: $28.3 \%$ versus $21.9 \%$, respectively.

- Alteration report:

- Male: none versus $25.0 \%$, respectively.

- Female: $12.5 \%$ versus none, respectively.

- Sexual activity decrease:

- Male: $32.4 \%$ versus $43.9 \%$, respectively.

- Female: $33.3 \%$ versus $25.0 \%$, respectively.

- Ejaculation or orgasm decrease:

- Male: $18.9 \%$ versus $30.0 \%$, respectively.

- Female: $33.3 \%$ versus $17.9 \%$, respectively.

For the other 2 items, the results were in the same line as above in the female patients, these items being not different between the treatment groups in the male patients:

- Inability to ejaculate or have an orgasm:

- Male: $18.9 \%$ and $15.4 \%$, respectively.

- Female: $31.0 \%$ versus $17.9 \%$, respectively.

- Erection difficulties:

- Male: $18.9 \%$ and $23.1 \%$, respectively.

- Female: $35.7 \%$ versus $21.4 \%$, respectively.

\section{CONCLUSION}

This international, multicentre, double-blind, randomised, 12-week study with 2 parallel groups aimed to assess the efficacy of agomelatine $25-50 \mathrm{mg} / \mathrm{day}$ compared to venlafaxine XR 75-150-225 mg/day in Asian non-depressed out-patients suffering from GAD. The study was prematurely stopped due to a sponsor's strategic decision. About one third of patients planned in the protocol were included before the end of the study. No statistically or clinically significant difference in the improvement of the anxiety symptoms assessed by the HAM-A total score was showed between the two treatment groups.

The safety profile of agomelatine $25-50 \mathrm{mg} / \mathrm{day}$ over the 14 -week treatment period was satisfactory and did not reveal any unexpected adverse events. The clinical acceptability of agomelatine was better than on venlafaxine, mainly for gastrointestinal and nervous system disorders.

Date of the report: 10 December 2014

Version of the report: Final version 